\title{
Triboson measurements, VV scattering and quartic gauge couplings with ATLAS and CMS
}

\author{
Riccardo BELLAN*广 \\ Università degli Studi di Torino and INFN \\ E-mail: riccardo.bellan@cern.ch
}

We present measurements of triboson production and vector boson scattering in pp collisions at 7 and $8 \mathrm{TeV}$ in the center-of-mass energy based on data recorded by the ATLAS and CMS detectors at the LHC in 2011 and 2012. The results are also interpreted in terms of constraints on anomalous quartic gauge couplings. In addition, we present studies of exclusive and quasiexclusive $\gamma \gamma \rightarrow W^{+} W^{-}$production p-p collisions at the LHC using the CMS detector, and new limits on anomalous quartic couplings.

XXII. International Workshop on Deep-Inelastic Scattering and Related Subjects,

28 April - 2 May 2014

Warsaw, Poland

\footnotetext{
* Speaker.

†n Behalf of the ATLAS and CMS collaborations.
} 


\section{Introduction}

Although the Standard Model (SM) of Particle Physics has been extensively tested at the colliders, proving to be accurate over many order of magnitude in the prediction of the process cross-sections and in the kinematic of the interactions, still some fundamental pieces need to be measured. The one question we had, does the Higgs boson exist?, has been now split into two paramount queries: is this the sole Higgs boson present in Nature? Is it really doing the Higgs boson job? To answer these questions, there are three main roads: keep investigating the high mass region, looking at the channels that brought to the Higgs boson discovery; measure with high precision (i.e., at percent level) the properties of the $125.5 \mathrm{GeV}$ boson; measure the Electroweak (EW) vector boson interactions, establishing whether this Higgs boson really can make unitaritypreserving the vector boson scattering (VBS) amplitudes at all energies.

The latter strategy is the topic of this note and aims at the measurement of the EW vector boson couplings with each other. Among the interesting interactions there are the triple gauge couplings (TGC), the not-yet-observed quartic gauge couplings (QGC) and the recently observed vector boson scattering processes. All these reactions foresee the production of one or many EW vector bosons, that with additional kinematic requirements on the hadronic activity topology (for VBS processes), marks a clear signature of the occurred interaction.

In these note I present the analyses at $\sqrt{s}=7-8 \mathrm{TeV}$ performed by the ATLAS [1] and CMS [2] experiments, installed along the Large Hadron Collider (LHC) ring. The results in this paper are divided in the following topics: mono-boson produced in VBS (sensitive to TGC), diboson produced in VBS (sensitive to the full set of EW vector boson self interaction diagrams, depending on the chosen final state), triboson final state not produced in VBS (sensitive to QGC). Results on the constraints of Anomalous Quartic Gauge Couplings (aQGC) are also presented, together with some hint of the projections of their measurements at 13-14 TeV in the centre-of-mass energy.

\section{Electroweak Production of $\mathrm{Z}$ in Association with two Forward/Backward Jets}

$Z$ vector boson can be produced through the fusion of two $W$ bosons, emitted by two quarks that then populate the forward-backward region, as it happens in VBS processes in which two bosons are produced after the proton-proton interaction. Therefore, both CMS and ATLAS analyses $[3,4]$ require a $Z$ produced in association with two tag jets well separated in rapidity, with high transverse momentum and large $m_{j j}$. ATLAS and CMS measure the cross section in two different fiducial regions. For $m_{j j}>120 \mathrm{GeV}$, CMS reports a measured cross section of $226 \pm$ 26 (stat) \pm 35 (sys) fb for $8 \mathrm{TeV}$ pp collisions. ATLAS reports a cross section of $54.7 \pm 4.6$ (stat) ${ }_{-10.4}^{+9.8}$ (sys) \pm 1.5 (lumi) fb for a less inclusive fiducial region, in particular for $m_{j j}>250 \mathrm{GeV}$ and a slightly different set of requirements on leptons and jets (for details see [4]). Figure 1 left panel displays the invariant mass of the dijet system, showing the evidence of the production of $Z$ boson through Electroweak processes.

Given the fact that the exchanged particle are colourless, a rapidity gap is expected in between the two tag jets. ATLAS exploited this feature to place a jet veto activity in between the two tag jets, while CMS studied the probability to have a third jet in the event. The right panel of Fig. 1 

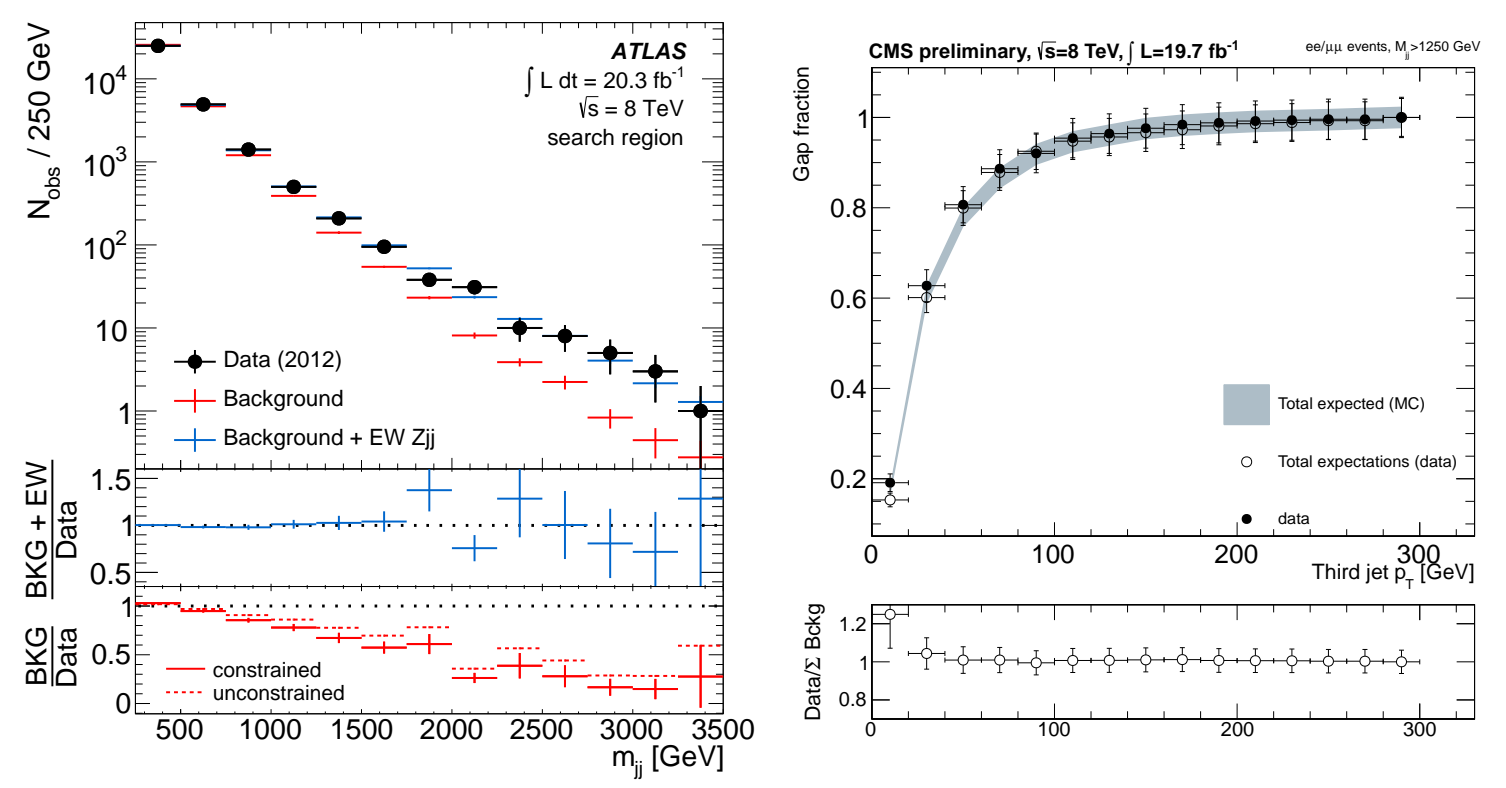

Figure 1: (Left) Dijet invariant mass distribution, from the $Z j j$ ATLAS analysis. The signal and (constrained) background templates are scaled to match the number of events obtained in the fit. The lowest panel shows the ratio of constrained and unconstrained background templates to the data; (right) Gap fraction for the $p_{T}$ of the third jet, from the $Z_{j j}$ CMS analysis. The observed fraction is compared to both data-driven and simulated predictions. The bottom panel shows the ratio between the observed data and the data-driven background prediction.

describes the probability of not having a third jet with a transverse momentum above the sliding $p_{T}$ cut represented on the abscissa.

\section{Diboson Production in Vector Boson Scattering}

The vector boson scattering is the key process to probe the Electroweak Symmetry Breaking (EWSB) and high energy vector boson scattering will play a central role both as a test of the nature of the newly discovered Higgs boson and as a model independent research of alternative theories able to explain the EWSB. If the discovered Higgs boson contributes fully to the EWSB, then most probably the interaction among longitudinal EW bosons would remain weak at high energy, otherwise if it is only partially responsible for the EWSB, then the vector boson interaction could get stronger at high energy in the centre of mass of the process.

Studies made by both ATLAS and CMS collaborations and several theorists indicate that an order of $100 \mathrm{fb}^{-1}$ at $\sqrt{s}=14 \mathrm{TeV}$ is needed to investigate sufficiently deeply these processes. However, with the already collected data at $\sqrt{s}=7-8 \mathrm{TeV}$, we can start to measure the cross sections of some processes dominated by vector boson interactions.

3.1 $p p \rightarrow W^{ \pm} W^{ \pm} j j \rightarrow l^{ \pm} l^{ \pm} v v j j$

The ATLAS collaboration reports the first evidence [5] for Electroweak $W^{ \pm} W^{ \pm} j j$ production, where both $W$ bosons decay leptonically $\left(W^{ \pm} \rightarrow \ell^{ \pm} v, \ell=e, \mu\right)$, using pp collision data at $\sqrt{s}=8 \mathrm{TeV}$. Cross sections are measured in two fiducial phase space regions ("fiducial regions") 


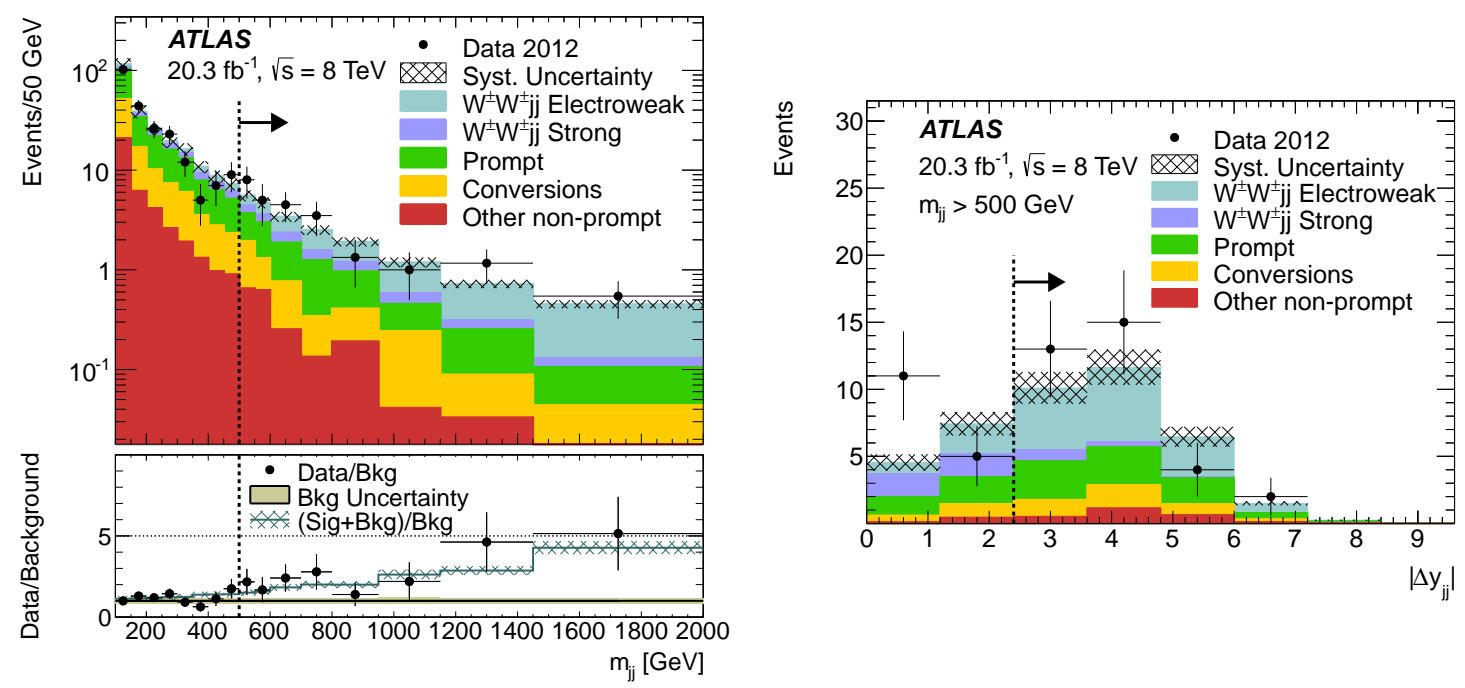

Figure 2: $W^{ \pm} W^{ \pm} j j$ ATLAS analysis. (Left) $m j j$ distribution for events passing all inclusive signal region selections except the $m_{j j}$ selection criteria. The black hatched band on the upper plot gives the systematic uncertainty on the total prediction, while the beige band on the lower plot gives the same uncertainty as a fraction of the total prediction; (right) $\left|\Delta y_{j j}\right|$ distribution for events passing all inclusive signal region selections. All three dilepton channels are summed. The $m_{j j}$ and $\left|\Delta y_{j j}\right|$ selections are indicated on each plot by a black dashed line. The contributions from Electroweak and Strong $W^{ \pm} W^{ \pm} j j$ production are normalised to the SM prediction.

respectively for the sum of Electroweak and Strong $W^{ \pm} W^{ \pm} j j$ production and for the Electroweak $W^{ \pm} W^{ \pm} j j$ process alone. The distinct experimental signature is two same electric charge leptons and two jets. Dominant backgrounds come from events with three or more leptons where one or more leptons are not reconstructed, asymmetric photon conversion in association with another lepton, and non-prompt production of same sign leptons.

Candidate $W^{ \pm} W^{ \pm} j j$ events are required to have exactly two leptons (electrons or muons) with the same electric charge and at least two jets with $p_{T}>30 \mathrm{GeV}$ and $|\eta|<4.5$. Three different final states are considered on the basis of the lepton flavor, namely $e^{ \pm} e^{ \pm}, e^{ \pm} \mu^{ \pm}$, and $\mu^{ \pm} \mu^{ \pm}$. The invariant mass of the two selected leptons must have $m_{\ell \ell}>20 \mathrm{GeV}$. Events are also required to have $E_{T}^{\text {miss }}>40 \mathrm{GeV}, m_{j j}>500 \mathrm{GeV}$, and not to contain a b-jet. The VBS enriched signal region in addition requires $\left|\Delta y_{j j}\right|>2.4$.

In Fig. 2 shows the distribution of $m_{j j}$ and $\left|\Delta y_{j j}\right|$ for the inclusive and the Electroweak regions, respectively. The combined significance over the background-only hypothesis is 4.5 standard deviations in the inclusive region and 3.6 standard deviations in the VBS region. The expected significance for a SM $W^{ \pm} W^{ \pm} j j$ signal is 3.4 standard deviations in the inclusive region and 2.8 in the VBS region. The measured cross sections in the fiducial regions are: $\sigma_{f i d}=2.1 \pm 0.5$ (stat) \pm 0.3 (sys) fb for the Strong and Electroweak $W^{ \pm} W^{ \pm} j j$ production, and $\sigma_{f i d}=1.3 \pm 0.4$ (stat) \pm 0.2 (sys) fb for the Electroweak $W^{ \pm} W^{ \pm} j j$ production. This analysis presents the first evidence of the Electroweak production of $W^{ \pm} W^{ \pm} j j$.

The measured cross section in the VBS fiducial region is used to set limits on aQGCs affecting vertexes with four interacting $W$ bosons. Following existing notations [6], the expected and 
observed $95 \%$ confidence intervals for the one-dimensional projection at $\alpha_{5 ; 4}=0$ is respectively found to be $-0.14<\alpha_{4}<0.16$ and $-0.23<\alpha_{5}<0.24$ compared to an expected $-0.10<\alpha_{4}<0.12$ and $-0.18<\alpha_{5}<0.20$. Over all, new physics scale in $W W W W$ coupling is set to be above $600 \mathrm{GeV}[7]$.

$3.2 p p \rightarrow p^{(*)} W^{ \pm} W^{\mp} p^{(*)} \rightarrow p^{(*)} l^{ \pm} l^{\mp} v v p^{(*)}$

The CMS collaboration analysed $\sqrt{s}=7 \mathrm{TeV}$ data searching for the production of opposite sign $W$ bosons, produced via $\gamma \gamma$ interaction [8]. The search is done looking at events with two different flavour opposite sign leptons with $p_{T}\left(\mu^{ \pm} e^{ \pm}\right)>30 \mathrm{GeV}$ and no other tracks coming from the two-leptons common vertex. This signature is also provided by quasi-exclusive ("inelastic" or "proton dissociative") production, where one or both incident protons dissociate into a low mass system that escapes detection, denoted as "*". The two-photons signal is therefore comprised of both the elastic and inelastic contributions.

In the full data-set of $L=5.05 \mathrm{fb}^{-1}$, two events pass all criteria, against a prediction of $0.84 \pm 0.13$ for the background events and $2.2 \pm 0.5$ for the signal. The measured cross-section, corresponding to a significance of 1.1 standard deviations, is $2.1_{-1.9}^{+3.1} \mathrm{fb}$, which translates into a cross section upper limit of $8.9 \mathrm{fb}$ at $95 \%$ confidence level (C.L.).

This analysis places also limits on anomalous $\gamma \gamma W W$ coupling, restricting the search to a region with $p_{T}\left(\mu^{ \pm} e^{ \pm}\right)>100 \mathrm{GeV}$. The model-independent $95 \%$ C.L. that has been found is: $\sigma\left(p p \rightarrow p^{(*)} W^{ \pm} W^{\mp} p^{(*)} \rightarrow p^{(*)} l^{ \pm} l^{\mp} v v p^{(*)}\right)<1.9$ fb. Limits on dimension-6 operators are displayed in Fig. 3.

\section{Triboson Production}

The structure of the triple and quartic gauge boson couplings emerges naturally from the SM non-Abelian $S U(2)_{L} \times U(1)_{Y}$ gauge symmetry. Together with the triple $W W \gamma$ and $W W Z$ gauge boson vertexes, the SM predicts the existence of the quartic $W W W W, W W Z Z, W W Z \gamma$ and $W W \gamma \gamma$ vertexes. The direct investigation of gauge boson self-interactions provides a crucial test on the gauge structure of the SM and is expected to play a significant role at LHC energies. The CMS collaboration presented a study of $W V \gamma$ production in semi-leptonic final state, which includes both $W(\rightarrow \ell v) W(\rightarrow j j) \gamma$ and $W(\rightarrow \ell v) Z(\rightarrow j j) \gamma$ processes [9], as they are indistinguishable due to the dijet mass resolution. The events are selected requiring only one high $p_{T}$ isolated lepton, $E_{T}^{\text {miss }}>35 \mathrm{GeV}$, and $M_{T}^{W}>30 \mathrm{GeV}$; two close-by non-b-tagged central jets and with $m_{j j}$ compatible with $m_{W / Z}$; finally, a central high $p_{T}$ photon. Figure 3 shows the distribution of the most sensitive variable $\left(p_{T}^{\gamma}\right)$ to possible $\mathrm{SM}$ deviations.

The $W W \gamma$ and $W Z \gamma$ cross section measurement in pp collisions at $\sqrt{s}=8 \mathrm{TeV}$ is not accessible with the data collected in 2012 by the CMS detector, it is only possible to set a one-sided upper limit on the cross section. An observed 95\% C.L. upper limit of $241 \mathrm{fb}$ is measured for the $W V \gamma$ cross section, which correspond to a cross section about 3.4 times larger than that predicted by the standard model. The expected limit is $309 \mathrm{fb}$ (4.4 times the SM cross section). 

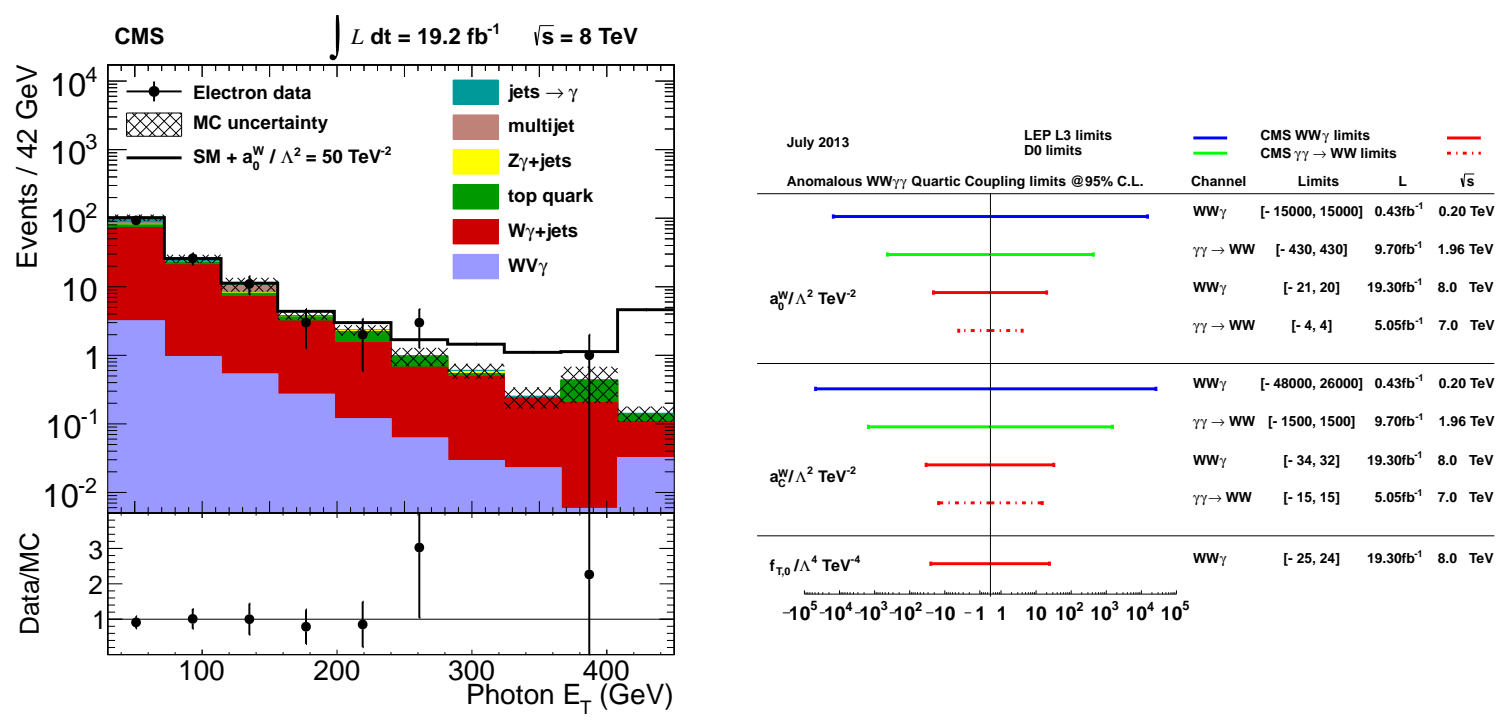

Figure 3: (Left) Comparison between predicted and observed photon $p_{T}$ distribution in electron channel. The rightmost bin includes the integral of events above $450 \mathrm{GeV}$ for each process; (right) CMS limits on quartic gauge couplings for diboson final states in scattering topologies and triboson final states. Limits are set in on dimension 6 and dimension 8 effective field theory operators.

\section{Conclusions and Outlook}

I presented the results obtained by the ATLAS and CMS collaboration on the measurement of the key feature of the Electroweak theory, i.e., the non-Abelian structure on which is founded. The first evidence of the Electroweak production of $W^{ \pm} W^{ \pm} j j$ is reported, together with a set of upper limits on the production cross-section of several processes able to intimately probe the Electroweak theory in general and the Electroweak Symmetry Breaking in particular. It is clear that although we discovered a Higgs boson, yet the comprehension of the Electroweak Symmetry Breaking is not completed, as several EW boson self-couplings still need to be observed. To exhaustively complete the picture, 100 to $300 \mathrm{fb}^{-1}$ of integrated luminosity of data collected at $\sqrt{s}=13-14 \mathrm{TeV}$ are needed, and on this respect, both ATLAS and CMS collaborations made preliminary studies on vector boson scattering processes $[10,11]$ with projections on the discovery potential of anomalous triple and quartic gauge couplings, in various models. The results, interpreted in terms of Effective Lagrangian to estimate the sensitivity to new physics, indicate that with an integrated luminosity of $300 \mathrm{fb}^{-1}$ the LHC experiments will be in the discovery regime for new physics manifesting with anomalies in the triple and quartic gauge couplings. This strongly indicates that the time of vector boson scattering and multiboson production is coming, and that it will be one of the hot topic at Run II of LHC.

\section{References}

[1] ATLAS Collaboration, The ATLAS experiment at the CERN LHC, JINST 3:S08003, 2008.

[2] CMS Collaboration, The CMS experiment at the CERN LHC, JINST 3:S08004, 2008. 
[3] CMS Collaboration, Measurement of pure electroweak production of a $Z$ boson in association with two forward/backward jets in proton-proton collisions at $8 \mathrm{TeV}$, CMS-PAS-FSQ-12-035.

[4] ATLAS Collaboration, Measurement of the electroweak production of dijets in association with a $Z$-boson and distributions sensitive to vector boson fusion in proton-proton collisions at $\sqrt{s}=8 \mathrm{TeV}$ using the ATLAS detector, JHEP 04 (2014) 031.

[5] ATLAS Collaboration, Evidence of the electroweak production of $W^{ \pm} W^{ \pm} j j$ in pp collisions at $\sqrt{s}=8 \mathrm{TeV}$ with the ATLAS detector, arXiv:1405.6241, submitted to PRL.

[6] A. Alboteanu, W. Kilian, J. Reuter, Resonances and Unitarity in Weak Boson Scattering at the LHC, JHEP 11 (2008) 010.

[7] J. Reuter, W. Kilian, M. Sekulla, Simplified Models for New Physics in Vector Boson Scattering Input for Snowmass 2013, arXiv:1307.8170.

[8] CMS Collaboration, Study of exclusive two-photon production of $W^{+} W$ in pp collisions at $\sqrt{s}=7 \mathrm{TeV}$ and constraints on anomalous quartic gauge couplings, JHEP 1307 (2013) 116.

[9] CMS Collaboration, $A$ Search for $W W \gamma$ and $W Z \gamma$ Production and Anomalous Quartic Gauge Couplings in pp Collisions at $\sqrt{s}=8 \mathrm{TeV}$, CMS-PAS-SMP-13-009 (submitted to Phys. Rev. D).

[10] ATLAS Collaboration, Studies of Vector Boson Scattering And Triboson Production with an Upgraded ATLAS Detector at a High-Luminosity LHC, ATL-PHYS-PUB-2013-006.

[11] CMS Collaboration, Vector Boson Scattering and Quartic Gauge Coupling Studies in WZ Production at $14 \mathrm{TeV}$, CMS-PAS-FTR-13-006. 